\title{
Single-Photon Detection using a Quantum-Dot-Gated Resonant RLC Circuit
}

\author{
Tyler Nickel, Yann Talhouarne, Andrew Prudhom, Richard Allenby, and Eric Gansen, ${ }^{\dagger}$ \\ Physics Department; University of Wisconsin-La Crosse, La Crosse, WI
}

\begin{abstract}
We report on a novel detection scheme that uses semiconductor quantum dots and electrical resonance to detect single photons of light. Here, a quantum-dot, optically gated field-effect transistor (QDOGFET) is used as the resistive element of a resonant RLC (resistor-inductor-capacitor) circuit. A photon is detected when it photocharges a quantum dot, thus modifying the resistance of the QDOGFET and altering the resonance condition of the surrounding circuit. Because the circuit functions as a bandpass filter, rejecting much of the electrical noise that can obscure weak photo-induced signals, the RLC detection scheme is sensitive enough to detect individual photons of light.
\end{abstract}

\section{Introduction}

The ability to detect single photons of light is fundamental to quantum information science and technology, is extremely useful for astronomical measurements, and may also lead to enhanced deep-space communication systems. In addition to being crucial measurement tools for experiments in quantum optics (Di Giuseppe et al., 2003; Waks et al., 2004; Waks et al., 2006; Achilles et al., 2006; Waks et al., 2006), single-photon detectors (SPDs) are needed for quantum communication systems based on quantum-key distribution (Brassard et al., 2000; Hiskett et al., 2006) and form the basis for certain strategies for quantum computing (Knill et al., 2001). In addition, arrays of SPDs are being developed to capture the faint images produced by telescopes (Romani et al., 1999), and they may also find use in the receivers of advanced laserbased (so called 'lasercom') systems that can transmit information at high speeds over interplanetary distances (Boroson et al., 2004; Mendenhall et al., 2007; Hemmati et al., 2007). For all of these applications, desired detector characteristics include high detection rates, low dark counts, high detection efficiency, low timing jitter, and photon-number resolving capability. In addition, SPDs should be compact, exhibit low power consumption, and be tolerant to changes in temperature and other environmental conditions.

In addition to detection mechanisms based on avalanche gain and low-temperature superconducting materials, another class of SPD that is being researched today makes use of semiconductor quantum dots (QDs). In one such device, referred to as a quantum dot, optically gated, field-effect transistor (QDOGFET) (Rowe et al., 2006), a layer of self-assembled QDs is embedded in a specially designed high-electron mobility transistor (HEMT). As illustrated in Fig. 1(a), the structure consists of alternating layers of GaAs and AlGaAs with a single layer of InGaAs QDs. Si delta doping provides excess electrons to the conduction band of the structure that accumulate at the GaAs/AlGaAs interface forming a two-dimensional electron gas (2DEG). The device is fabricated by depositing electrical contacts, denoted as the source and drain, on the semiconductor structure; by etching a mesa between the contacts to channel the 2DEG; and by

\footnotetext{
${ }^{\dagger}$ The authors would like to acknowledge M. A. Rowe, S. M. Etzel, S. W. Nam, and R. P. Mirin of the Quantum Electronics and Photonics Division of the National Institute of Standards and Technology (NIST) in Boulder, CO for their contributions to this work as well as the Wisconsin Space Grant Consortium for its financial support.
} 
depositing a thin ribbon of platinum across the channel mesa. The platinum ribbon is used to gate the transistor and is thin enough (4 nm thick) to partially transmit light. The area where photons are detected is defined by the gated portion of the 2DEG channel. The detection area is typically about four square micrometers in size and contains approximately 2000 QDs.

The basic mechanism that makes QDOGFETs photosensitive is described in Figure 1(a). During operation, electrical current flows through the 2DEG channel while a negative bias is applied to the gate. The key to detecting photons with this structure is that the resistance of the 2DEG depends strongly on the electric field produced by the gate. A photon produces a response when it charges a QD by exciting an electron-hole pair in the GaAs absorption region. Due to the internal electric field, the hole is directed to the QDs, where it is trapped, while the electron joins the 2DEG. The electric field associated with the charged QD combines with the electric field produced by the gate modifying the total field 'seen' by the current. Consequently, the absorbed photon causes a small change in the transistor resistance, which has been traditionally read out by monitoring the current (Rowe et al., 2006; Gansen et al., 2007a; Gansen et al., 2007b; Rowe et al., 2008; Rowe et al., 2010; Gansen et al., 2013). The resistance change persists for as long at the hole is confined to the dot, and it is the photoconductive gain associated with this process that makes QDOGFETs extremely photosensitive.
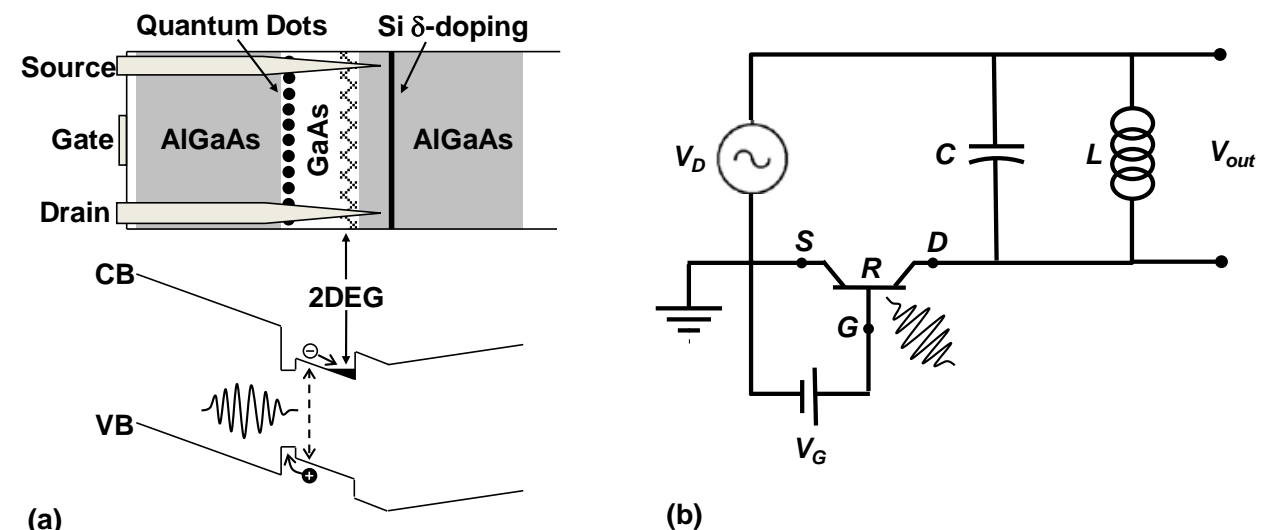

(b)

Figure 1. (a) Schematic diagram of the composition and band structure of the QDOGFET single-photon detector. CB and VB denote the conduction band and valence band, respectively, and 2DEG denotes the two-dimensional electron gas. (b) QD-gated resonant RLC detection circuitry that utilizes a QDOGFET as the resistive and photosensitive element. $V_{D}$ and $V_{G}$ denote the drive and gate voltages, respectively.

The photoresponse of QDOGFETs and similarly designed structures (Shields et al., 2000; Shields et al., 2001; Kardynal et al., 2004; Kardynal et al., 2006; Kardynal et al., 2007) has been studied extensively at cryogenic temperatures $(4 \mathrm{~K}-77 \mathrm{~K})$. They have been shown to exhibit photon-number-resolving capabilities (Gansen et al., 2007b; Kardynal et al., 2007; Rowe et al., 2008), detection rates as high as $400 \mathrm{kHz}$ (Kardynal et al., 2004; Gansen et al., 2013), and when properly designed, high internal quantum efficiency (Rowe et al., 
2006; Gansen et al., 2007). However, while persistent photoconductivity lasting for hours has been demonstrated in transistors for temperatures as high as $150 \mathrm{~K}$ (Finley et al., 1998), demonstrations of single-photon detection using QDOGFETs and similar structures have been limited to cryogenic temperatures $(<77 \mathrm{~K})$ due to electrical noise (Shields et al., 2001; Gansen et al., 2013). Electrical noise is the random fluctuation in current and voltage that is present in all electrical circuits. It is a common problem in detectors as it can obscure weak photo-induced signals. Electrical noise in two-dimensional electron systems has been studied extensively due to its impact on high-speed HEMTs (Kirtley et al., 1988; Hofman et al., 1990; Paransin et al., 1990; Chen et al., 2000), Hall bar structures (Müller et al., 2006 and reference therein), and SPDs (Rowe et al., 2010; Gansen et al., 2013). Studies on QDOGFETs have shown that the power spectral density of the noise in such structures is typically $1 / f$ in nature (i.e. inversely proportional to frequency) and increases with temperature (Rowe et al., 2010; Gansen et al., 2013).

Here, we report on a novel detection scheme that promises to reduce the impact of electrical noise in QD-based detectors. In this scheme, QDs are embedded in the resistive element of a resonant RLC (resistor/inductor/capacitor) circuit, as shown schematically in Fig. 1(b). A QDOGFET functions as the resistive element and the absorber for the detection system. When a photon is absorbed in the QDOGFET, it modifies the 2DEG resistance by charging a QD and subsequently modifies the resonant behavior of the circuit. The advantage the resonant detection circuitry has over traditional detection electronics is that the resonant nature of the circuit prohibits electrical noise at off-resonant frequencies from contaminating the responses produced by photons. In this way, the circuit functions as a bandpass filter, only allowing signals with frequencies near the resonant frequency to contribute the output signal, $V_{\text {out }}$. Consequently, the resonant detection circuitry is expected to provide enhanced sensitivity over traditional read out schemes that provide no such noise discrimination.

The sensitivity of the detection scheme is derived from the photoconductive gain provided by the persistent nature of the QDOGFET's response coupled with the resonant nature of the surrounding detection circuitry. Other forms of resonance have been shown to be sensitive mechanisms for detecting individual photons, as exemplified by QD resonant tunneling diodes (Blakesley et al., 2005). In these devices, photocharged QDs are used to shift the energies of discrete levels required for resonant tunneling through a double-barrier junction. The resonance, in this case, is characteristic of the semiconductor structure itself and is not easily modeled. By contrast, the resonance conditions for RLC circuits are well understood and easily engineered.

In this work, we demonstrate the ability of a QD-gated resonant RLC circuit to detect single photons of light and discuss how it works. First, we describe the detection mechanism in detail by presenting the results of mathematical simulations and experimental measurements of the electrical characteristics of the circuitry. We then demonstrate the photosensitivity of the detection system by presenting the results of measurements where we illuminate the active area 
of the QDOGFET with highly attenuated laser pulses. Finally, we characterize the performance of the system by performing statistical analysis of the optical data. We show that the device can detect single photons of light with a signal-to-noise ratio of 2.7:1 at an operating temperature of $6 \mathrm{~K}$.

\section{Detection System and Principles of Operation}

As illustrated schematically in Fig. 1, the detection circuitry consists of a QDOGFET wired in series with the parallel combination of an inductor, $L$, and a capacitor, $C$. The circuit is driven with a sinusoidal bias voltage, $V_{D}$. The transistor contacts and 2DEG channel are the dominant resistive elements in the circuit and are represented in the schematic by total resistance, $R$. The conductivity of the QDOGFET channel is variable and controlled by the gate voltage, $V_{G}$.

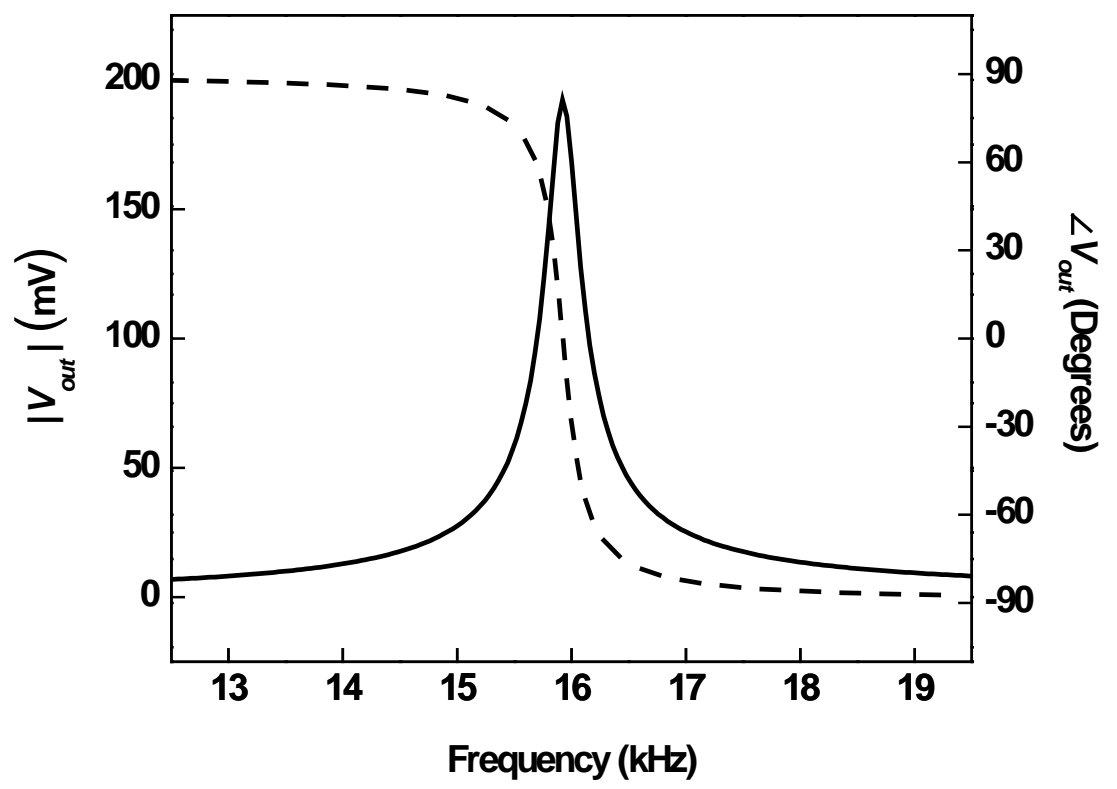

Figure 2. Mathematical simulation of the amplitude, $\left|V_{\text {out }}\right|$, and phase, $\angle V_{\text {out }}$, of the output signal as a function of the driving frequency for $L=1 \mathrm{mH}, C=100 \mathrm{nF}, R=30 \mathrm{k} \Omega$, and $0.7 \Omega$ parasitic resistance in the inductor and capacitor branches of the circuit. The drive voltage was taken to be $1 \mathrm{~V}_{\mathrm{pp}}$.

In Fig. 2 we show the results of simulations that demonstrate the resonant nature of the detection circuit. From basic circuit analysis, the characteristic parameters of the ideal circuit (which assumes no resistance in the inductor and capacitor branches) are easily determined and are given by: amplitude $\left|V_{o}\right|=\left|V_{D}\right|$; resonant frequency $f_{o}=(2 \pi \sqrt{L C})^{-1}$; and bandwidth $\Delta f=(2 \pi R C)^{-1}$. For a circuit containing components with values $L=1 \mathrm{mH}, C=100 \mathrm{nF}$ and $R=30 \mathrm{k} \Omega$, the ideal model yields $\left|V_{o}\right|=1 \mathrm{~V}, f_{o}=15.9 \mathrm{kHz}$ and $\Delta f=53 \mathrm{~Hz}$. However, parasitic resistance in the branches of the circuits will modify the resonant conditions. The simulation shown in Fig. 2 includes parasitic resistances of $0.7 \Omega$ in both the capacitive and inductive branches of the circuit. Such resistance is reasonable for actual circuit components, and as we 
will show, provides good agreement between simulation and experimental data. Parasitic resistance does not modify the resonant frequency of the circuit considerably, but it does reduce the magnitude and quality factor of the resonance. In the simulation, approximately $20 \%$ of the $1 \mathrm{~V}_{\mathrm{pp}}$ (peak-to-peak voltage) drive voltage is passed by the circuit on resonance, and the bandwidth of the circuit is $\sim 370 \mathrm{~Hz}$ (as defined by the full-width at the $\sqrt{2}^{-1}$ of the maximum points).

When a photogenerated hole is trapped in a QD, the charged dot screens the gate field changing the resistance of the transistor channel. The simulation shown in Fig. 3 illustrates the effect of the photo-induced change in $R$ on the resonant peak. In this simulation it was assumed that a hole trapped in a QD changes the channel resistance of the QDOGFET by $2 \Omega$. The result indicates that the dominate effect of the resistance change is an increase in the magnitude of the resonance. Consequently, the largest photoresponse should be achieved when the circuit is driven at its resonant frequency.

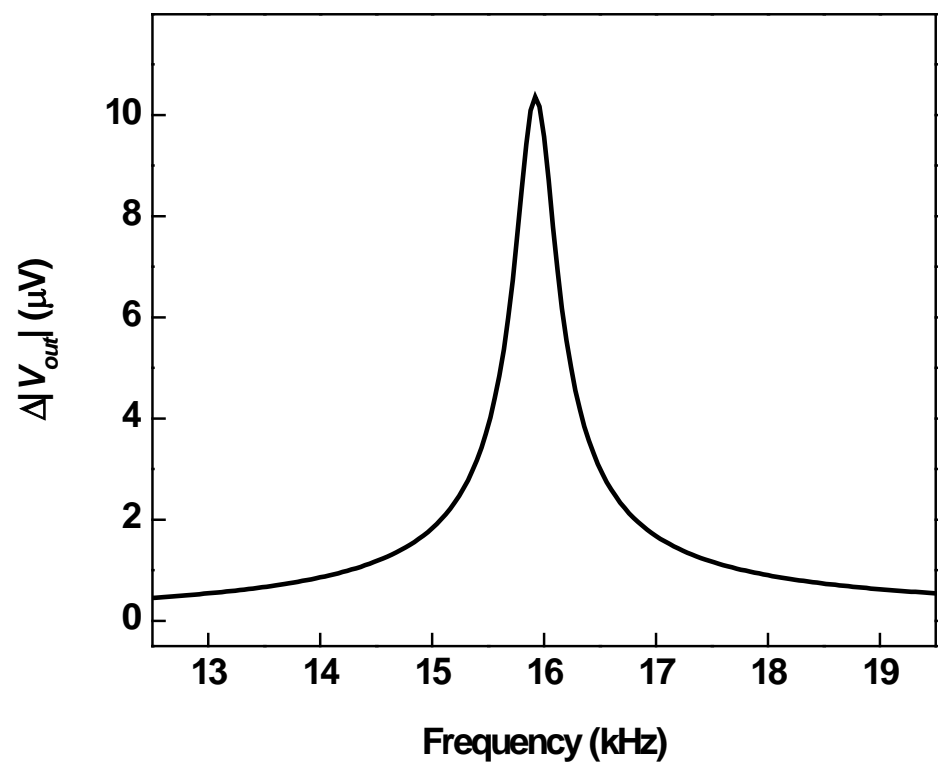

Figure 3. Mathematical simulation of the change in $\left|V_{\text {out }}\right|$ caused by reducing the QDOGFET channel resistance by $2 \Omega$. All other circuit parameters are the same as indicated in Fig. 2.

The simulations are to be compared with the experimental data shown in Fig. 4, which demonstrates the electrical characteristics of a QD-gated resonant RLC circuit. The structural details of the specific QDOGFET used in the circuit can be found in Rowe et al., 2006 and Gansen et al., 2007a. The circuit components (capacitor, inductor, and QDOGFET) were mounted on the cold stage of a liquid helium cryostat and cooled to $6 \mathrm{~K}$. The inductor and capacitor used in the circuit exhibited room temperature values of $L=1 \mathrm{mH}$ and $C=100 \mathrm{nF}$; however, these values undoubtedly changed at the operating temperature. Fig. 4(a) shows the dependence of $\left|V_{\text {out }}\right|$ on the drive frequency for a drive voltage of $1 \mathrm{~V}_{\mathrm{pp}}$ and for $V_{G}=0 \mathrm{~V}$. The maximum output voltage is similar to that obtained in the 
simulation $\left(V_{o}=200 \mathrm{mV}\right.$ pp), while the experimental peak is broader $(\Delta f=715 \mathrm{~Hz}$ ) and shifted to a higher central frequency $\left(f_{o}=22.4 \mathrm{kHz}\right)$.

To maximize the photosensitivity of the detection system, it is important to bias the gate of the QDOGFET such that $V_{\text {out }}$ is sensitive to changes in the gate field. In Fig. 4(b) we show how the magnitude of the resonance depends on the gate voltage. Optimal photoresponse is achieved for gate voltages where the slope of the curve is largest. It has been shown and substantiated with experiments (Rowe et al., 2006; Gansen et al., 2007a; Gansen et al., 2007b; Rowe et al., 2008; Rowe et al., 2010; Gansen et al., 2013) that the change in $V_{G}$ caused by the trapping of $N$ holes in the QD layer is given by

$$
\Delta V_{G}=\frac{e W}{\varepsilon^{\prime} A} N
$$

where $e$ is the elementary charge, $W$ is the distance between the gate contact and the QD layer, $\varepsilon^{\prime}$ is the electric permittivity of the material, and $A$ is the transistor active area. Consequently, the photo-induced change in the output signal is given by

$$
\Delta\left|V_{\text {out }}\right|=M \frac{e W}{\varepsilon^{\prime} A} N
$$

where $M=d V_{\text {out }} / d V_{G}$ represents the slope of the curve shown in Fig. 4(b). Given the electrical properties of the circuit and the geometry and composition of the QDOGFET, each photon is expected to modulate the output voltage by $\Delta\left|V_{\text {out }}\right|=10.5 \mu \mathrm{V}_{\mathrm{pp}}$ for $V_{G}=-0.5 \mathrm{~V}$.
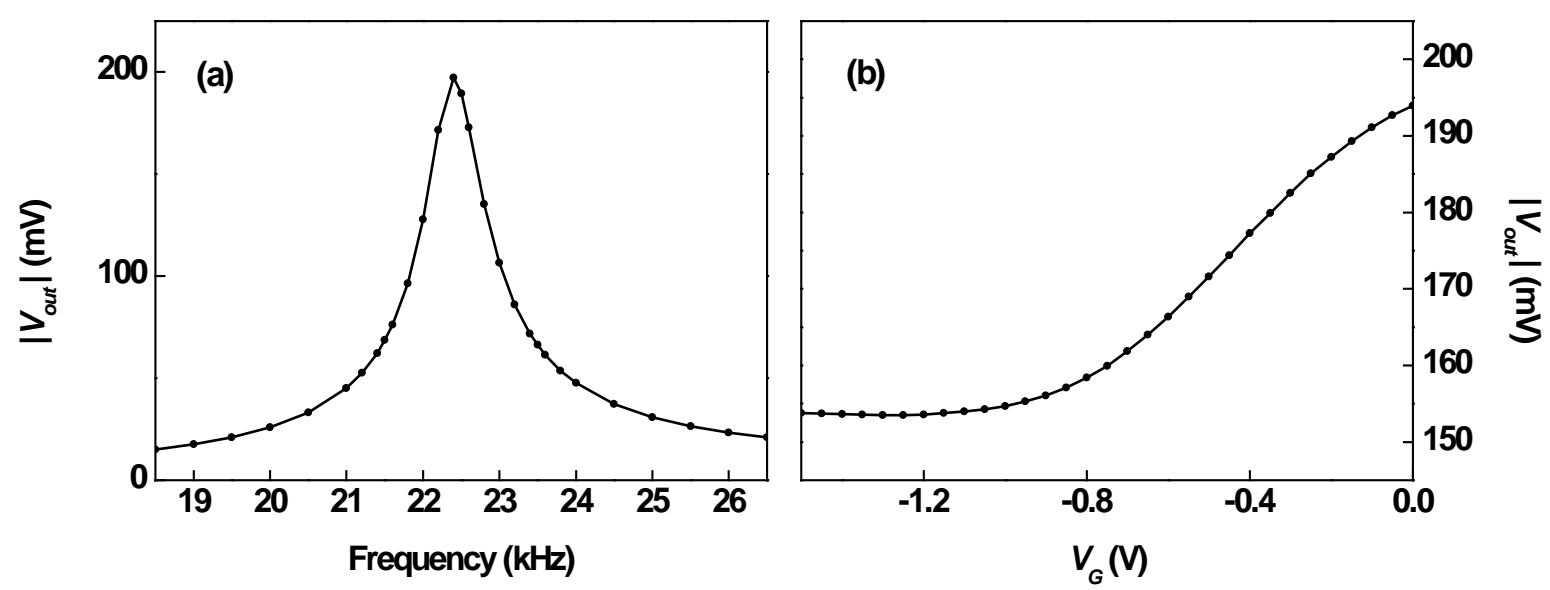

Figure 4. Experimental measurements of (a) $\left|V_{\text {out }}\right|$ as a function of the driving frequency for $V_{G}=0 \mathrm{~V}$ and (b) $\left|V_{\text {out }}\right|$ as a function of $V_{G}$ with the circuit driven at its resonance frequency. In both (a) and (b) the drive voltage was $1 \mathrm{~V}_{\mathrm{pp}}$. 


\section{Experimental Demonstration of Single-Photon Detection}

A schematic of the measurement system used to test the photoresponse of the QD-gated resonant RLC circuit is shown in Fig. 5. The AC output voltage of the circuit was amplified by a preamplifier and then sent to a lock-in amplifier that was referenced to the drive frequency. The analog output signal from the lock-in amplifier was subsequently sent through a low-pass filter with a cut-off frequency of $1 \mathrm{kHz}$ and converted to a digital signal for collection by a computer. Taking into account the voltage gain provided by the added detection electronics, the amplified signal captured by the computer is given by $V_{c}=G\left|V_{\text {out }}\right|$, where $G=667$ is the total gain provided by the amplifiers. With this amplification in place, theory (Eq. [2]) predicts that each photon should produce a $7.0 \mathrm{mV}$ change in $V_{c}$ when the circuit is driven at resonance.

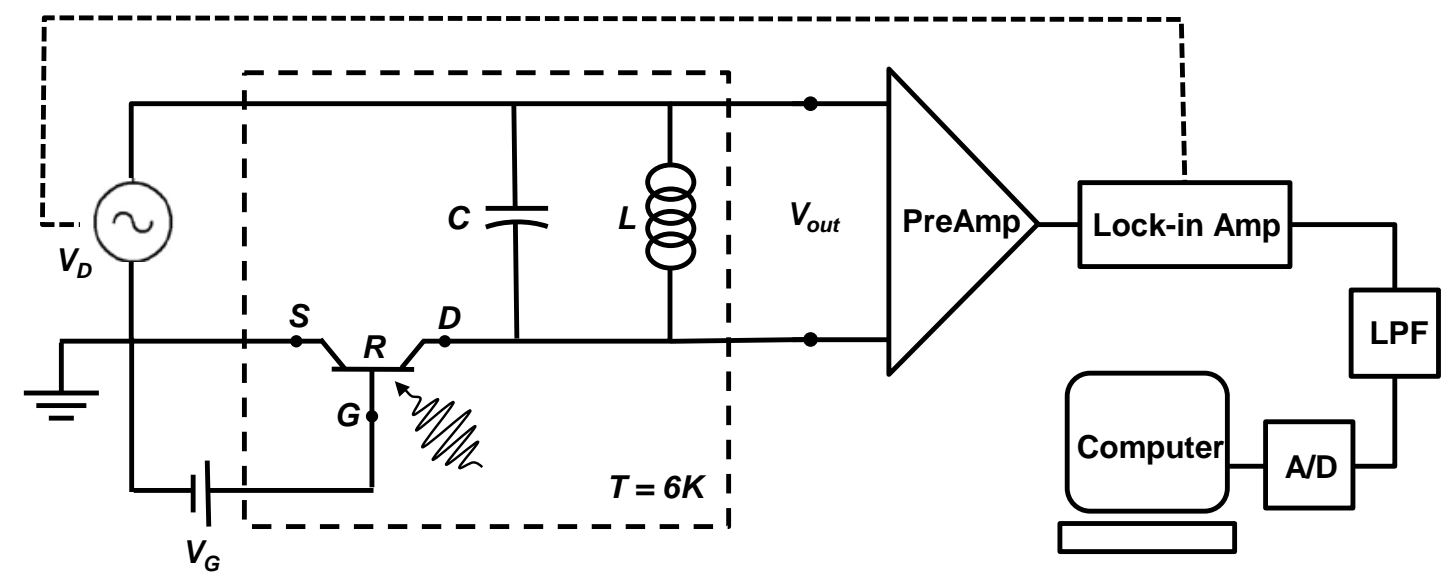

Figure 5. Schematic diagram of the electronics used to test the photosensitivity of the QD-gated resonant RLC detection circuitry. The total voltage gain of the preamplifier and lock-in amplifier combination was 667, and the cut-off frequency of the low-pass filter (LPF) was $1 \mathrm{kHz}$. A computer with an analogto-digital (A/D) converter card was used to collect and store the amplified signals from the detection circuitry which was housed in a liquid helium cryostat (represented by dashed box).

The detection system was tested by illuminating the active area of the QDOGFET with a 1-Hz train of laser pulses from a diode laser that were properly tuned to be absorbed in the GaAs absorption layer of the transistor. In these measurements, the RLC circuit was cooled to $6 \mathrm{~K}$, and the 300-ns-long laser pulses were attenuated such that on average less than one photon was detected per pulse. During illumination, a bias of $-0.5 \mathrm{~V}$ was applied to the gate to maximize the photoresponse of the detection system; however, $500 \mathrm{~ms}$ after each pulse, the gate voltage was temporarily raised to $+1.0 \mathrm{~V}$ for $1 \mathrm{~ms}$ to discharge the dots. Here, the electrical reset pulse flooded the QDs with conduction band electrons which recombined with trapped holes (Gansen et al., 2007a; Rowe et al., 2008).

In Fig. 6, we show the results of measurements where we illuminated the QDOGFET with 3000 laser pulses and monitored the magnitude of the system's response to each pulse of light. A 
typical trace showing the system's response to a pulse of light arriving at $t=0.31 \mathrm{~s}$ is shown in Fig. 6(a). The response of the system is characterized by a persistent increase in the amplified output voltage where the rise time ( $13 \mathrm{~ms}$ ) of the signal is limited by the passband of the RLC circuit and the cut-off frequency of the low-pass filter.

A histogram of the 3000 responses is shown in Fig. 6(b). Here, we averaged the amplified output signal over 500- $\mu$ s intervals leading up to and following a 30 -ms window surrounding the arrival time of each pulse. We then subtracted the two averaged values to determine the magnitude of the response produced by each pulse. A histogram acquired without illumination is also shown for comparison. In the absence of photons, the histogram is characterized by a single Gaussian-shaped peak centered at zero volts. The width of the peak is representative of the electrical noise in the system. With illumination, the central Gaussian peak is reduced by comparison (indicating fewer occurrences of zero detected photons), and an additional shoulder is observed in the histogram comprised of non-zero photocounts.
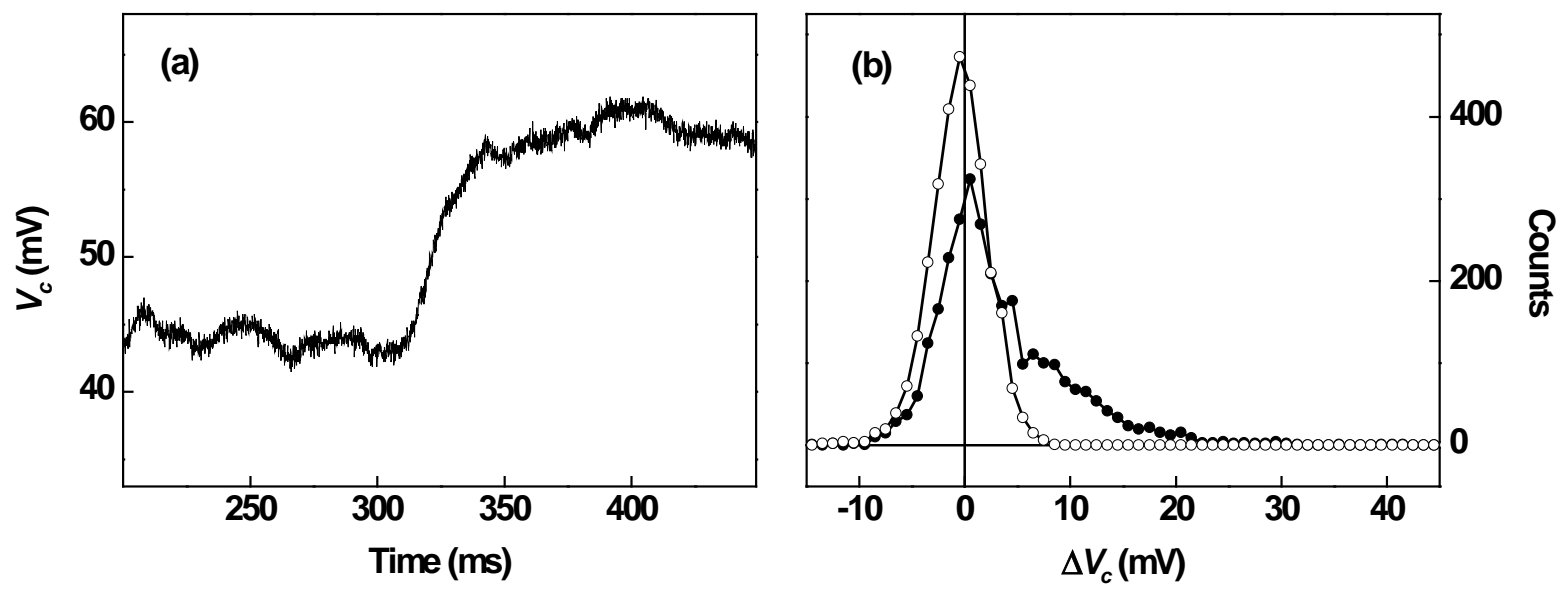

Figure 6. (a) Single-shot measurement of $V_{c}$ responding to a laser pulse arriving at $t=0.31 \mathrm{~ms}$. The detection circuit was driven at its resonant frequency $\left(f_{D}=f_{o}=22.5 \mathrm{kHz}\right)$ with a drive voltage of $1 \mathrm{~V}_{\mathrm{pp}}$. The gate bias was set at $V_{G}=-0.5 \mathrm{~V}$ during the arrival of the light pulses. $500 \mathrm{~ms}$ after each pulse it was raised to $V_{G}=1 \mathrm{~V}$ for $1 \mathrm{~ms}$ to empty the QDs. (b) Histograms of binned signal changes, $\Delta V_{c}$, with (solid circles) and without (open circles) illumination.

The mean number of photons detected per pulse, $\lambda$, and the average magnitude of the response per photon can be determined by conducting a statistical analysis of the histograms shown in Fig. 6(b). The probability that $N$ photons are detected for any given light pulse from a Poisson source is given by $P(N)=\left(\lambda^{N} / N !\right) e^{-\lambda}$. The central Gaussian peak (associated with zero photons) in the histogram acquired with illumination has $69 \%$ as many counts as the Gaussian peak acquired without illumination. As a result, the mean number of photons detected per pulse is estimated from $P(0)=e^{-\lambda}$ to be $\lambda=0.38$. It follows directly that $P(1)=0.26$ and $P(2)=0.05$. Consequently, most of the photocounts observed in the histogram with illumination are produced by single photons of light with fewer coming from higher numbers of photons. With 
illumination, the average signal produced is $3.3 \mathrm{mV}$, which when divided by $\lambda$ yields an average amplified response of $\Delta V_{c}=8.7 \mathrm{mV}$ per photon. This value agrees quite well with the amplified response predicted by theory. It is most likely an overestimate of the single-photon response caused by the absorption of photons in the gated portions of the channel mesa as the capture of photo-generated holes in QDs near but beyond the edges of the gate contact has been shown to contribute photo-induced signals in QDOGFETs (Gansen et al., 2007a).

The single-photon sensitivity of the detection system is apparent by comparing the average response produced by individual photons to the random signal changes produced by electrical noise. The standard deviation of the histogram acquired without illumination is $3.3 \mathrm{mV}$, which is $37 \%$ the size of the average response produce by a single photon. As a result, most photoevents can be clearly distinguished from noise, which is the defining characteristic of a single-photon detector.

\section{Conclusions}

We have demonstrated the ability of a QD-gated resonant RLC circuit to detect single photons of light. We described the detection mechanism using mathematical simulations and experimental measurements of the circuit's electrical characteristics, and we tested the detection system by performing optical measurements. We found that the single-photon response of the system is consistent with that predicted by theory and is clearly distinguishable from the electrical noise produced in the circuitry. Future work will include optimizing the detection circuitry to improve the sensitivity of the system, demonstrating photon-number-discriminating detection by incorporating a number-resolving QDOGET into the circuit, and investigating the temperature and speed limitations of the system.

\section{References}

[1] Achilles, D., Silberhorn, C., and Walmsley, I. A., "Direct, loss-tolerant characterization of nonclassical photon statistics," Phys. Rev. Lett. 97, 43602 (2006).

[2] Blakesley, J. C., See, P., Shields, A. J., Kardynal, B. E., Atkinson, P., Farrer, I., and Ritchie, D. A., "Efficient single photon detection by quantum dot resonant tunneling diodes," Phys. Rev. Lett. 94, 67401 (2005).

[3] Boroson, D. M., Bondurant, R. S., and Scozzafava, J. J., "Overview of high rate deep space laser communications options," in Free-Space Laser Communication Technologies XVI, G. S. Mecherle, C. Y. Young, and J. S. Stryjewsjki, eds., Proc. SPIE 5338, 37-49 (2004).

[4] Brassard, G., Lütkenhaus, N., Mor, T., and Sanders, B. C., "Limitations on practical quantum cryptography," Phys. Rev. Lett. 85, 1330-1333 (2000).

[5] Chen, Y., VanVliet, C. M., Larkins, G. L., and Morkoç, H., ”Generation-Recombination Noise in Nongated and Gated $\mathrm{Al}_{\mathrm{x}} \mathrm{Ga}_{1-\mathrm{x}} \mathrm{As} / \mathrm{GaAs}$ TEGFETs in the Range $1 \mathrm{~Hz}$ to $1 \mathrm{MHz}$," IEEE Trans. Electron Devices 47, 2045-2053 (2000).

[6] Di Giuseppe, G., Atatüre, M., Shaw, M. D., Sergienko, A. V., Saleh, B. E. A., Teich, M. C., Miller, A. J., Nam, S. W., and Martinis, J., "Direct observation of photon pairs at a single output of a beam-splitter interferometer," Phys. Rev. A 68, 63817 (2003).

[7] Finley, J. J., Skalitz, M., Arzberger, M., Zrenner, A., Böhm, G., and Abstreiter, G., "Electrical detection of optically induced charge storage in self-assembled InAs quantum dots,” Appl. Phys. Lett. 73, 2618 (1998). 
[8] (a) Gansen, E. J., Rowe, M. A., Greene, M. B., Rosenberg, D., Harvey, T. E., Su, M. Y., Hadfield, R. H., Nam, S. W., and Mirin, R. P., "Operational Analysis of a Quantum Dot Optically Gated Field-Effect Transistor as a Single-Photon Detector;” IEEE. J. Sel. Topics Quantum Electron. 13, 1-11 (2007).

[9] (b) Gansen, E. J., Rowe, M. A., Greene, M. B., Rosenberg, D., Harvey, T. E., Su, M. Y., Hadfield, R. H., Nam, S. W., and Mirin, R. P., "Photon-Number-Discriminating Detection using a Quantum-Dot, Optically Gated, Field-Effect Transistor,” Nature Photonics 1, 585-588 (2007).

[10] Gansen, E. J., Rowe, M. A., Harrington, S. D., Nehls, J. M., Etzel, S. M., Nam, S. W., and Mirin, R. P., "Temperature Dependence of the Single-Photon Sensitivity of a Quantum Dot, Optically Gated, FieldEffect Transistor,” J. Appl. Phys. 114, 093103 (2013).

[11] Hemmati, H., Biswas, A., and Boroson, D., "Prospects for Improvement of Interplanetary Laser Communication Data Rates by 30 dB,” Proc. IEEE 95, 2082-2092 (2007).

[12]Hiskett, P. A., Rosenberg, D., Peterson, C. G., Hughes, R. J., Nam, S. W., Lita, A. E., Miller, A. J., and Nordholt, J. E., “Long-distance quantum key distribution in optical fibre,” New J. of Phys. 8, 193 (2006).

[13]Hofman, F., Zijlstra, R. J. J., and Bettencourt de Freitas, J. M., "Voltage Noise in an $\mathrm{Al}_{\mathrm{x}} \mathrm{Ga}_{1-\mathrm{x}} \mathrm{As}-\mathrm{GaAs}$ Heterostructure,” J. Appl. Phys. 67, 2482-2487 (1990).

[14]Kardynal, B. E., Hees, S. S., Shields, A. J., Nicoll, C., Farrer, I., and Ritchie, D. A., "Photon number resolving detector based on a quantum dot field effect transistor,” Appl. Phys. Lett. 90, 181114 (2007).

[15] Kardynal, B. E., Shields, A. J., Beattie, N. S., Farrer, I., Cooper, K., and Ritchie, D. A., "Low-noise photon counting with a radio-frequency quantum-dot field-effect transistor,” Appl. Phys. Lett. 84, 419-421 (2004).

[16] Kardynal, B. E., Shields, A. J., Farrer, I., Cooper, K., and Ritchie, D. A., "Single electron dynamics in a quantum dot field effect transistor,” Appl. Phys. Lett. 89, 113503 (2006).

[17] Kirtley, J. R., Theis, T. N., Mooney, P. M., and Wright, S. L., “Noise Spectroscopy of Deep Level (DX) Centers in GaAs- $\mathrm{Al}_{\mathrm{x}} \mathrm{Ga}_{1-\mathrm{x}}$ As Heterostructures,” J. Appl. Phys. 63, 1541-1547 (1988).

[18] Knill, E., Laflamme, R., and Milburn, G. J., “A scheme for efficient quantum computation with linear optics,” Nature 409, 46-52 (2001).

[19] Mendenhall, J. A., Candell, L. M., Hopman, P. J., Zogbi, G., Boroson, D. M., Caplan, D. O., Digenis, C. J., Hearn, D. R., and Shoup, R. C., "Design of an Optical Photon Counting Array Receiver System for DeepSpace Communications,” Proc. IEEE 95, 2059-2069 (2007).

[20] Müller, J., Li, Y., von Molnár, S., Ohno, Y., and Ohno, H., "Single-Electron Switching in $\mathrm{Al}_{\mathrm{x}} \mathrm{Ga}_{1-\mathrm{x}} \mathrm{As} / \mathrm{GaAs}$ Hall Devices,” Phys. Rev. B 74, 125310 (2006).

[21] Peransin, J. M., Vignaud, P., Rigaud, D., and Vandamme, L. K. J., “1/f Noise in MODFET’s at Low Drain Bias,” IEEE Trans. Electron Devices 37, 2250-2253 (1990).

[22] Romani, R. W., Miller, A. J., Cabrera, B., Figueroa-Feliciano, E., and Nam, S. W., “First Astronomical Application of a Cryogenic Transition Edge Sensor Spectrophotometer,” Astrophys. J. 521, L153-L156 (1999).

[23] Rowe, M. A., Gansen, E. J., Greene, M. B., Hadfield, R. H., Harvey, T. E., Su, M. Y., Nam, S. W., and Mirin, R. P., "Single-photon detection using a quantum dot optically gated field-effect transistor with high internal quantum efficiency,” Appl. Phys. Lett. 89, 253505 (2006).

[24] Rowe, M. A., Gansen, E. J., Greene, M. B., Rosenberg, D., Harvey, T. E., Su, M. Y., Hadfield, R. H., Nam, S. W., and Mirin, R. P., "Designing High Electron Mobility Transistor Heterostructures with Quantum Dots for Efficient, Number-Resolving Photon Detection,” J. Vac. Sci. Technol. B 26, 1174-1177 (2008).

[25] Rowe, M. A., Salley, G. M., Gansen, E. J., Etzel, S. M., Nam, S. W., and Mirin, R. P., “Analysis of photoconductive gain as it applies to single-photon detection,” J. Appl. Phys. 107, 63110 (2010). 
[26] Shields, A. J., O’Sullivan, M. P., Farrer, I., Ritchie, D. A., Hogg, R. A., Leadbeater, M. L., Norman, C. E., and Pepper, M., "Detection of single photons using a field-effect transistor gated by a layer of quantum dots,” Appl. Phys. Lett. 76, 3673-3675 (2000).

[27] Shields, A. J., O’Sullivan, M. P., Farrer, I., Ritchie, D. A., Leadbeater, M. L., Patel, N. K., Hogg, R. A., Norman, C. E., Curson, N. J., and Pepper, M., "Single photon detection with a quantum dot transistor," Jpn. J. Appl. Phys. 40, 2058-2064 (2001).

[28] Waks, E., Diamanti, E., Sanders, B. C., Bartlett, S. D., and Yamamoto, Y., "Direct observations of nonclassical photon statistics in parametric down-conversion,” Phys. Rev. Lett. 92, 113602 (2004).

[29] Waks, E., Diamanti, E., and Yamamoto, Y., “Generation of photon number states,” New Journ. Phys. 8, 4-8 (2006).

[30]Waks, E., Sanders, B. C., Diamanti, E., and Yamamoto, Y., "Highly nonclassical photon statistics in parametric down-conversion," Phys. Rev. A 73, 33814 (2006). 